\title{
TRACKING CONTROL OF PARABOLIC SYSTEMS
}

\author{
Luciano Pandolfi* \\ Politecnico di Torino, Dip. di Matematica \\ Lucipan@polito.it \\ Enrico Priola \\ Università di Torino, Dip. di Matematica \\ priola@dm.unito,it
}

\begin{abstract}
We consider the tracking problem for parabolic systems with boundary control. Assuming that the reference signal is bounded and measurable, we prove various regularity results as well representation formulas for the optimal control and the optimal trajectory.
\end{abstract}

Keywords: Regulator problem, distributed systems, boundary control

\section{Introduction and Preliminaries}

The quadratic regulator problem for distributed parameter systems was analyzed in the monographs $[1,5]$, in particular the (time and space) regularity properties of the Riccati equations, arising in boundary control of PDEs, is deeply studied in [5]. However variants of the quadratic regulator problem like the tracking and cheap control did not receive much attention in the boundary control case.

The aim of this paper is to partially fill this gap, by investigating the tracking problem. This consists in finding a control $v$ to force the output $z$ of a given system to follow a desired reference signal $y$; we refer to $[3,8]$ for an introduction to this problem in finite dimensions. We obtain regularity results for the optimal control $v$ as well as useful representation formulas involving $v$ and the optimal trajectory $w$, see in

\footnotetext{
* The research of both the authors was supported in part by the Italian Ministero dell'Università e della Ricerca Scientifica e Tecnologica. It fits the program of GNAMPA.
} 
particular Theorems 8, 10 and 13. These are extensions of known finite dimensional formulas (see [3]) to the present boundary control case.

Since the presence of the reference signal $y$ entails a lack of regularity in the solution and a different form of the optimal control, our theorems are not contained in the monograph [5]. Moreover the results which are presented here will be applied to the study of the cheap control problem in a forthcoming paper.

The system that we consider is described by

$$
\dot{w}(t)=A w(t)+B v(t), \quad t \in(0, T), \quad w(0)=w_{0},
$$

where $A$ generates an exponentially stable holomorphic semigroup on a Hilbert space $X$ and $v \in L^{2}(0, T ; X)$. Exponential stability is assumed only in order to simplify the notations. The operator $B$ takes values in $\left(\operatorname{dom} A^{*}\right)^{\prime}$. Here $A^{*}$ denotes the adjoint of $A$ and $\left(\operatorname{dom} A^{*}\right)^{\prime}$ stands for the topological dual of $\operatorname{dom} A^{*}$ (the space $\operatorname{dom} A^{*}$ is endowed with the graph norm); see [5]for more details as well as for several applications of (1). We assume that for some $\gamma \in[0,1)$,

$$
D=(-A)^{-\gamma} B \in \mathcal{L}(U, X),
$$

i.e. $D$ is a bounded linear operator from $U$ to $X$, where $U$ is a second Hilbert space (if $A$ is not stable then the notation $(-A)^{\gamma}$ is to be replaced by $(-A-r I)^{\gamma}$ with $r$ large enough). Note that (2) is equivalent to $B \in \mathcal{L}\left(U,\left[\operatorname{dom}\left(-A^{*}\right)^{\gamma}\right]^{\prime}\right)$ and implies that, for some $\sigma$ and $M>0$,

$$
\left\|B^{*} e^{A^{*} t}\right\| \leq \frac{M e^{-\sigma t}}{t^{\gamma}}, t>0 .
$$

The tracking problem is the following:

$$
\begin{aligned}
& \min _{v} J_{\alpha}\left(w_{0} ; v\right), \quad J_{\alpha}\left(w_{0} ; v\right)=\int_{0}^{T}\left\{\|z(t)-y(t)\|^{2}+\alpha\|v(t)\|^{2}\right\} d t, \\
& z(t)=z\left(t ; w_{0}, v\right)=C w\left(t ; w_{0}, v\right),
\end{aligned}
$$

where $\alpha>0$ is fixed, $y$ is a prescribed reference signal and $w\left(t ; w_{0}, v\right)$ denotes the solution to (1); further $C$ is a linear and bounded operator from $X$ to a third Hilbert space $Y$. The cheap control problem consisting in studying the limit for $\alpha \rightarrow 0^{+}$will be studied in the sequel. The standing assumption on $y$ is that it is measurable and bounded, i.e. $y \in L^{\infty}(0, T ; Y)$.

Let us introduce the following operators

$$
\begin{aligned}
L v(t) & =\int_{0}^{t} e^{A(t-s)} B v(s) d s, \quad \Lambda=C L \\
L^{*} w(t) & =B^{*} \int_{t}^{T} e^{A^{*}(s-t)} w(s) d s, \quad \Lambda^{*}=L^{*} C^{*} ; \quad \Gamma w_{0}(t)=C e^{A t} w_{0},
\end{aligned}
$$


$w_{0} \in X$. The properties of these operators have been precisely studied in [5]. Moreover we recall from [5, p. 13 and p. 23]:

Theorem 1 Let $\gamma \in[0,1)$ as in (2). We have:

$$
L \in \mathcal{L}\left(L^{2}(0, T ; U), L^{2}\left(0, T ; \operatorname{dom}(-A)^{1-\gamma}\right)\right)
$$

and $L \in \mathcal{L}\left(L^{\infty}(0, T ; U), C\left([0, T] ; \operatorname{dom}(-A)^{\theta}\right)\right)$ for every $0 \leq \theta<1-\gamma$

We observe that we are in the second smoothing case studied in [5]so that we can freely use all the regularity results in that book, which concern the solutions of the Riccati equation and the operators $L$ and $\Lambda$. Using a result in [6], we can improve Lemma 1 as follows (as usual, $C^{\sigma}$ denotes the space of Hölder continuous functions):

Theorem 2 For any $\theta \in[0,1-\gamma)$, we have

$$
L \in \mathcal{L}\left(L^{\infty}(0, T ; U), C^{1-\gamma-\theta}\left([0, T] ; \operatorname{dom}(-A)^{\theta}\right) .\right.
$$

In particular $L \in \mathcal{L}\left(L^{\infty}(0, T ; U), C^{1-\gamma}([0, T] ; X)\right.$.

Proof We write:

$$
L v(t)=\int_{0}^{t} e^{A(t-s)} B v(s) d s=(-A)^{\gamma} \int_{0}^{t} e^{A(t-s)} D v(s) d s,
$$

see (2). Now recall that in Proposition 4.2.2 in [6], see also Sec. 2.2.2 in $[6]$, it is proved that the operator $R$,

$$
R f(t)=\int_{0}^{t} e^{A(t-s)} f(s) d s
$$

belongs to $\mathcal{L}\left(L^{\infty}(0, T ; X), C^{1-\alpha}\left([0, T] ; \operatorname{dom}(-A)^{\alpha}\right)\right.$, for any $\alpha \in(0,1)$. Remark that this implies also that $R \in \mathcal{L}\left(L^{\infty}(0, T ; X), C^{1-\alpha}([0, T] ; X)\right)$, for any $\alpha \in(0,1)$. Applying this result to $L$ we get the assertion.

\section{The Tracking Problem}

Let us consider (4). The existence of the optimal control $v_{\alpha}$ is clear,

$$
v_{\alpha}=\left(\alpha+\Lambda^{*} \Lambda\right)^{-1} \Lambda^{*}\left(y-\Gamma w_{0}\right) .
$$

Let $w_{\alpha}$ be the state produced by $v_{\alpha}$, i.e. the solution of Eq. (1) when $v=v_{\alpha}$. Let moreover $z_{\alpha}=C w_{\alpha}=\Lambda v_{\alpha}+\Gamma w_{0}$ be the corresponding output. We easily obtain from (5) a second representation formula for the optimal control:

$$
v_{\alpha}=\frac{1}{\alpha} \Lambda^{*}\left[y-z_{\alpha}\right] .
$$


Using Young inequalities, we see that $\Lambda^{*}\left(y-\Gamma w_{0}\right)$ is continuous on $[0, T]$ and $\left\|\Lambda^{*}\left(y-\Gamma w_{0}\right)(t)\right\| \leq M(T-t)^{1-\gamma}$. Moreover from [5, Theorem 1.4.4.4] $\left(\alpha+\Lambda^{*} \Lambda\right)^{-1}$ is boundedly invertible on $C_{\gamma}(0, T ; U)$, where

$$
C_{\gamma}(0, T ; U)=\left\{f \in C[0, T) ; U \text { uch that } \sup _{t \in(0, T)}(T-t)^{\gamma}|f(t)|<+\infty\right\} .
$$

Hence we have that $v_{\alpha}$ is continuous on $[0, T)$. In addition the usual bootstrap argument, based on Young inequalities, shows:

THEOREM 3 The optimal control $v_{\alpha}$ is continuous on $[0, T]$. Hence, also $w_{\alpha}(t)$ and $z_{\alpha}(t)$ are continuous too. Moreover, $\left\|v_{\alpha}(t)\right\|=\mathrm{O}(T-t)^{1-\gamma}$.

Combining Lemmas 2 and 3 we obtain:

THEOREM 4 The function $w_{\alpha}$ is Hölder continuous on every compact interval contained in $(0, T]$ with values in $X$. The Hölder exponent is $1-\gamma$.

\section{Proof From}

$$
w_{\alpha}(t)=e^{A t} w_{0}+\int_{0}^{t} e^{A(t-s)} B v_{\alpha}(s) d s,
$$

we need only to prove Hölder continuity of the integral (since the first addendum is continuously differentiable for $t>0$, because $e^{A t}$ is a holomorphic semigroup). To this end it is enough to apply Lemma 2.

In the next result, we give two representations of the minimum value of the cost.

THEOREM 5 We have:

$$
J_{\alpha}\left(w_{0} ; v_{\alpha}\right)=\left\langle y-\Gamma w_{0}, y-z_{\alpha}\right\rangle=\left\langle y-\Gamma w_{0}, \alpha\left(\alpha+\Lambda \Lambda^{*}\right)^{-1}\left(y-\Gamma w_{0}\right)\right\rangle .
$$

Proof In the following computation, $\tilde{y}=y-\Gamma w_{0}$ and norm and inner product are in $L^{2}$. We note that

$$
\begin{aligned}
& \left\|\Lambda v_{\alpha}-\tilde{y}\right\|^{2}+\alpha\left\|v_{\alpha}\right\|^{2}=\left\langle\Lambda v_{\alpha}, \Lambda v_{\alpha}\right\rangle-2\left\langle\Lambda v_{\alpha}, \tilde{y}\right\rangle+\|\tilde{y}\|^{2}+\alpha\left\|v_{\alpha}\right\|^{2} \\
& =\left\langle v_{\alpha}, \Lambda^{*} \Lambda\left(\alpha+\Lambda^{*} \Lambda\right)^{-1} \Lambda^{*} \tilde{y}\right\rangle-2\left\langle v_{\alpha}, \Lambda^{*} \tilde{y}\right\rangle+\|\tilde{y}\|^{2}+\alpha\left\|v_{\alpha}\right\|^{2} \\
& =\left\langle v_{\alpha}, \Lambda^{*} \tilde{y}-\alpha\left(\alpha+\Lambda^{*} \Lambda\right)^{-1} \Lambda^{*} \tilde{y}\right\rangle-2\left\langle v_{\alpha}, \Lambda^{*} \tilde{y}\right\rangle+\|\tilde{y}\|^{2}+\alpha\left\|v_{\alpha}\right\|^{2} \\
& =-\left\langle v_{\alpha}, \Lambda^{*} \tilde{y}\right\rangle+\|\tilde{y}\|^{2}=\left\langle\tilde{y}, \tilde{y}-\Lambda v_{\alpha}\right\rangle
\end{aligned}
$$

since

$$
\tilde{y}-\Lambda v_{\alpha}=y-z_{\alpha} .
$$

This is the first representation. The second representation is obtained from here, since

$$
\Lambda\left(\alpha+\Lambda^{*} \Lambda\right)^{-1} \Lambda^{*}=\left(\alpha+\Lambda \Lambda^{*}\right)^{-1} \Lambda \Lambda^{*} .
$$


The proof is complete.

We introduce now the explicit form of $(6)$ :

$$
v_{\alpha}(t)=\frac{1}{\alpha} \int_{t}^{T} B^{*} e^{A^{*}(s-t)} C^{*}\left[y(s)-z_{\alpha}(s)\right] d s=-\frac{1}{\alpha} B^{*} p_{\alpha}(t)
$$

where

$$
p_{\alpha}(t)=-\int_{t}^{T} e^{A^{*}(s-t)} C^{*}\left[y(s)-z_{\alpha}(s)\right] d s .
$$

We note that $p_{\alpha} \in C([0, T] ; X)$, since $C$ is a bounded operator and

$$
\|p(t)\| \leq M(T-t)^{(1-\gamma)}
$$

For $t=0$ we have the equality

$$
p_{\alpha}(0)=-\int_{0}^{T} e^{A^{*} s} C^{*}\left[y(s)-z_{\alpha}(s)\right] d s=-\Gamma^{*}\left(y-z_{\alpha}\right)
$$

so that we find a third representation for the optimal cost,

$$
J_{\alpha}\left(w_{0} ; v_{\alpha}\right)=\left\langle y, y-z_{\alpha}\right\rangle_{L^{2}(0, T ; Y)}+\left\langle w_{0}, p_{\alpha}(0)\right\rangle_{X} .
$$

The function $p_{\alpha}$ is the weak solution of

$$
\dot{p}=-A^{*} p-C^{*}\left[C w_{\alpha}-y\right], \quad p(T)=0 .
$$

We have the condition $p(T)=0$ since the final value of $w$ is not penalized. In this way we arrive at the usual hamiltonian system

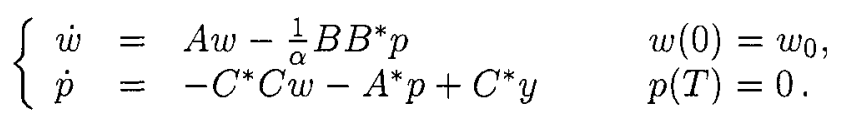

The functions $p_{\alpha}$ and $w_{\alpha}$ solve (10) in a weak sense. We improve the regularity of $p_{\alpha}$ in the next Lemma.

THEOREM 6 We have $p_{\alpha} \in C^{1-\theta}\left([0, T] ; \operatorname{dom}\left(-A^{*}\right)^{\theta}\right)$, for every $0<\theta<$ 1 , and $p_{\alpha} \in L^{2}\left(0, T ; \operatorname{dom} A^{*}\right)$.

Proof The first assertion follows from (8), taking into account that the function $C^{*}\left[C w_{\alpha}-y\right]$ is bounded and applying Proposition 4.2.2 in [6]. The second statement can be proved as in [4], see also [5, p. 4].

Let us consider now the special but important case when $y$ is Hölder continuous. Using [6, Theorem 4.3.4] and [7, Theorem 3.5.], we get:

THEOREM 7 If there exists $\eta \in(0,1), \eta \leq 1-\gamma$, s.t. $y \in C^{\eta}([\epsilon, T] ; Y)$ for every $\epsilon>0$, then $p_{\alpha}$ is continuously differentiable, and the derivative is Hölder continuous too, i.e., $p_{\alpha} \in C^{1+\eta}([\epsilon, T], X)$, for every $\epsilon>0$. 
In the next result we consider the regularity of the $\operatorname{map} B^{*} p_{\alpha}=-\alpha v_{\alpha}$. Theorem 8 Let $\eta \in(0,1)$ and $y \in C^{\eta}([\epsilon, T] ; Y)$, for any $\epsilon>0$. The functions $B^{*} p_{\alpha}$ (and so also $v_{\alpha}$ ) is Hölder continuous on compact intervals contained in $(0, T]$, of exponent $\min \{\eta, 1-\gamma\}$.

Proof Let $f=-C^{*}\left[C w_{\alpha}-y\right]$. The function $f$ is bounded. Now fix $\epsilon>0$ and take $t^{\prime \prime}>t^{\prime} \geq \epsilon$. We obtain

$$
\left[p_{\alpha}\left(t^{\prime \prime}\right)-p_{\alpha}\left(t^{\prime}\right)\right]=\int_{0}^{T-t^{\prime \prime}} e^{A^{*} s} f\left(s+t^{\prime \prime}\right) d s-\int_{0}^{T-t^{\prime}} e^{A^{*} s} f\left(s+t^{\prime}\right) d s .
$$

Hence,

$$
\begin{aligned}
& {\left[B^{*} p\left(t^{\prime \prime}\right)-B^{*} p\left(t^{\prime}\right)\right]=B^{*} \int_{T-t^{\prime \prime}}^{T-t^{\prime}} e^{A^{*} s} f\left(s+t^{\prime}\right) d s} \\
& +B^{*} \int_{0}^{T-t^{\prime \prime}} e^{A^{*} s}\left[f\left(s+t^{\prime \prime}\right)-f\left(s+t^{\prime}\right)\right] d s
\end{aligned}
$$

The function $f$ is bounded so that the first addendum is less then

$$
\int_{T-t^{\prime \prime}}^{T-t^{\prime}} \frac{M_{0}}{s^{\gamma}} d s \leq M_{1}\left\{\left(T-t^{\prime}\right)^{1-\gamma}-\left(T-t^{\prime \prime}\right)^{1-\gamma}\right\} \leq M\left(t^{\prime \prime}-t^{\prime}\right)^{(1-\gamma)} .
$$

The second integral is the sum of the following two terms:

$$
\begin{aligned}
& B^{*} \int_{0}^{T-t^{\prime \prime}} e^{A^{*} s} C^{*}\left[y\left(s+t^{\prime}\right)-y\left(s+t^{\prime \prime}\right)\right] d s \\
& B^{*} \int_{0}^{T-t^{\prime \prime}} e^{A^{*} s} C^{*} C\left[w_{\alpha}\left(s+t^{\prime \prime}\right)-w_{\alpha}\left(s+t^{\prime}\right)\right] d s .
\end{aligned}
$$

Hölder continuity of $y$ and condition (3) imply that the norm of (11) is less then $M_{\epsilon}\left[t^{\prime \prime}-t^{\prime}\right]^{\eta}$. The second integral is treated analogously, and we get a similar estimate, with exponent $1-\gamma$ on every interval $[\epsilon, T]$, $\epsilon>0$, see Lemma 4 .

A further regularity result that is needed below is as follows:

THEOREM 9 Let $x_{0} \in \operatorname{dom} A^{*}$. The function:

$$
t \longrightarrow\left\langle x_{0}, \int_{0}^{t} e^{A(t-s)} B v_{\alpha}(s) d s\right\rangle=\left\langle\left(-A^{*}\right)^{\gamma} x_{0}, \int_{0}^{t} e^{A(t-s)} D v_{\alpha}(s) d s\right\rangle,
$$

is differentiable on $[0, T]$ and moreover

$$
\begin{aligned}
& \frac{d}{d t}\left\langle x_{0}, \int_{0}^{t} e^{A(t-s)} B v_{\alpha}(s) d s\right\rangle \\
& =\left\langle\left(-A^{*}\right)^{\gamma} x_{0}, D v_{\alpha}(t)\right\rangle+\left\langle A^{*} x_{0}, \int_{0}^{t} e^{A(t-r)} B v_{\alpha}(r) d r\right\rangle
\end{aligned}
$$


Proof First recall that the function $t \rightarrow D v_{\alpha}(t)$ is continuous on $[0, T]$. Then let $\delta>0$; because the semigroup is holomorphic, we have

$$
\begin{aligned}
& \frac{d}{d t}\left\langle x_{0}, \int_{0}^{t-\delta} e^{A(t-s)} B v_{\alpha}(s) d s\right\rangle=\frac{d}{d t}\left\langle\left(-A^{*}\right)^{\gamma} x_{0}, \int_{0}^{t-\delta} e^{A(t-s)} D v_{\alpha}(s) d s\right\rangle \\
& =\left\langle\left(-A^{*}\right)^{\gamma} x_{0}, e^{A \delta} D v_{\alpha}(t-\delta)\right\rangle+\left\langle\left(-A^{*}\right)^{\gamma} x_{0}, A \int_{0}^{t-\delta} e^{A(t-s)} D v_{\alpha}(s) d s\right\rangle \\
& =\left\langle\left(-A^{*}\right)^{\gamma} x_{0}, e^{A \delta} D v_{\alpha}(t-\delta)\right\rangle+\left\langle A^{*} x_{0},(-A)^{\gamma} \int_{0}^{t-\delta} e^{A(t-s)} D v_{\alpha}(s) d s\right\rangle .
\end{aligned}
$$

We see from here that

$\left\|\frac{d}{d t}\left\langle x_{0}, \int_{0}^{t-\delta} e^{A(t-s)} B v_{\alpha}(s) d s\right\rangle\right\| \leq M\left\|v_{\alpha}\right\|_{\infty}\left\{\left\|\left(-A^{*}\right)^{\gamma} x_{0}\right\|+\left\|A^{*} x_{0}\right\|\right\}$.

Thanks to this estimate, we can use dominated convergence theorem and we can pass to the limit for $\delta \rightarrow 0^{+}$in the following equality:

$$
\begin{aligned}
& \left\langle\left(-A^{*}\right)^{\gamma} x_{0}, \int_{0}^{t-\delta} e^{A(t-s)} D v_{\alpha}(s) d s\right\rangle=\int_{\delta}^{t-\delta}\left\{\left\langle\left(-A^{*}\right)^{\gamma} x_{0}, e^{A \delta} D v_{\alpha}(s-\delta)\right\rangle\right. \\
& \left.+\left\langle A^{*} x_{0}, \int_{0}^{s-\delta} e^{A(s-r)} B v_{\alpha}(r) d r\right\rangle\right\} d s .
\end{aligned}
$$

We differentiate both sides of the resulting equality and we get:

$$
\begin{aligned}
& \frac{d}{d t}\left\langle x_{0}, \int_{0}^{t} e^{A(t-s)} B v_{\alpha}(s) d s\right\rangle \\
& =\left\langle\left(-A^{*}\right)^{\gamma} x_{0}, D v_{\alpha}(t)\right\rangle+\left\langle A^{*} x_{0}, \int_{0}^{t} e^{A(t-r)} B v_{\alpha}(r) d r\right\rangle .
\end{aligned}
$$

The proof is complete.

If $y=0$ it is well known that the optimal control can be put in feedback form. This is not possible if $y \neq 0$ since at a given time $t$ the future values of $y$, which affect the optimal control, are unknown. Infact we have:

THEOREM 10 We have $p_{\alpha}(t)=P_{\alpha}(t) w_{\alpha}(t)+d_{\alpha}(t)$ where $P_{\alpha}$ solves the Riccati differential equation

$$
\begin{aligned}
& \left\langle\frac{d}{d t} P_{\alpha}(t) x, y\right\rangle=-\left\langle P_{\alpha}(t) A x, y\right\rangle-\left\langle P_{\alpha}(t) x, A y\right\rangle \\
& -\langle C x, C y\rangle+\frac{1}{\alpha}\left\langle B^{*} P_{\alpha}(t) x, B^{*} P_{\alpha}(t) y\right\rangle, \quad P_{\alpha}(T)=0 .
\end{aligned}
$$


Here $x$ and $y$ are arbitrary elements in $\operatorname{dom} A$. The function $d_{\alpha}$ is continuous on $[0, T]$ and is zero for $t=T$. It depends on $y$ but not on $w_{0}$ and it is given by (here $\left.\left(\Lambda_{t} u\right)(r)=\int_{t}^{r} C e^{A(r-s)} B u(s) d s\right)$

$$
\begin{aligned}
& d_{\alpha}(t)=-\int_{t}^{T} e^{A^{*}(s-t)} C^{*} y(s) d s \\
& +\int_{t}^{T} e^{A^{*}(s-t)} C^{*} C \int_{t}^{s} e^{A(s-r)} B\left[\left(\alpha+\Lambda_{t} \Lambda_{t}^{*}\right)^{-1} \Lambda_{t}^{*} y\right](r) d r d s .
\end{aligned}
$$

Proof The operator valued function $P_{\alpha}(t)$ is the solution of the usual Riccati equation. For each $x \in X, P_{\alpha}(\cdot) x$ is continuous on $[0, T]$ and it is zero for $t=T$. Moreover, it is differentiable on $(0, T)$, with continuous and bounded derivative in closed subintervals, see Theorem [5, p. 19-20]. In order to prove the theorem, it is sufficient to show that the continuous function $d_{\alpha}(t)=p_{\alpha}(t)-P_{\alpha}(t) w_{\alpha}(t)$ only depends on the tracking signal $y$. We introduce the functions $v^{+}\left(s ; t, x_{0}\right)$ and $w^{+}\left(s ; t, x_{0}\right), x_{0} \in X$, the solutions of the optimization problem under study, in the case that $y=0$ and with initial condition $x_{0}$ at time $t$ instead then 0 . Hence, $v^{+}=-(\alpha+$ $\left.\Lambda_{t}^{*} \Lambda_{t}\right)^{-1} \Lambda_{t}^{*} \Gamma_{t} x_{0}$, where $\Gamma_{t} x_{0}(s)=C e^{(s-t) A} x_{0}$ (note that $v^{+}$depends on $\alpha)$. Moreover, we use the following representation formula for $P_{\alpha}(t)$ :

$$
P_{\alpha}(t) x=\int_{t}^{T} e^{A^{*}(r-t)} C^{*} C w^{+}(r ; t, x) d r .
$$

Hence,

$$
\begin{aligned}
& p_{\alpha}(t)-P_{\alpha}(t) w_{\alpha}(t)=-\int_{t}^{T} e^{A^{*}(s-t)} C^{*} y(s) d s \\
& +\int_{t}^{T} e^{A^{*}(s-t)} C^{*} C\left[w_{\alpha}\left(s ; 0, w_{0}\right)-w^{+}\left(s ; t, w_{\alpha}\left(t ; 0, w_{0}\right)\right)\right] d s .
\end{aligned}
$$

For clarity, in this formula we indicated explicitly the initial time and initial value of $w_{\alpha}$. Now we use dynamic programming: the optimal control on $[t, T]$, with initial condition $w_{\alpha}\left(t ; 0, w_{0}\right)$ is the restriction to $[t, T]$ of $v_{\alpha}$. This holds for every given reference signal $y$. Hence,

$$
\begin{aligned}
& w_{\alpha}\left(s ; 0, w_{0}\right)-w^{+}\left(s ; t, w_{\alpha}\left(t ; 0, w_{0}\right)\right) \\
& =w_{\alpha}\left(s ; t, w_{\alpha}\left(t ; 0, w_{0}\right)\right)-w^{+}\left(s ; t, w_{\alpha}\left(t ; 0, w_{0}\right)\right) \\
& =\left(e^{A(s-t)}\left\{w_{\alpha}\left(t ; 0, w_{0}\right)-w_{\alpha}\left(t ; 0, w_{0}\right)\right]\right) \\
& \quad+\int_{t}^{s} e^{A(s-r)} B\left[v_{\alpha}(r)-v^{+}(r)\right] d r
\end{aligned}
$$




$$
\begin{aligned}
& =\int_{t}^{s} e^{A(s-r)} B\left\{\left(\alpha+\Lambda_{t}^{*} \Lambda_{t}\right)^{-1}\left[\Lambda_{t}^{*}\left(y-\Gamma_{t} w_{0}\right)+\Lambda_{t}^{*}\left(\Gamma_{t} w_{0}\right)\right\}\right\}(r) d r \\
& =\int_{t}^{s} e^{A(s-r)} B\left[\left(\alpha+\Lambda_{t}^{*} \Lambda_{t}\right)^{-1} \Lambda_{t}^{*} y\right](r) d r
\end{aligned}
$$

as wanted.

In fact, in finite dimensions $d_{\alpha}$ solves

$$
\dot{d}_{\alpha}(t)=-\left(A^{*}-\frac{1}{\alpha} P_{\alpha}(t) B B^{*}\right) d_{\alpha}(t)+C^{*} y(t), \quad d_{\alpha}(T)=0 .
$$

We will show that an analogous result holds in general. We prove first:

THEOREM 11 We have, for every $x \in \operatorname{dom} A$,

$$
\begin{aligned}
& \frac{d}{d t}\left\langle x, d_{\alpha}(t)\right\rangle \\
& =\left\langle-A x, d_{\alpha}(t)\right\rangle+\frac{1}{\alpha}\left\langle B^{*} P_{\alpha}(t) x, B^{*} d_{\alpha}(t)\right\rangle+\left\langle x, C^{*} y(t)\right\rangle, d_{\alpha}(T)=0 .
\end{aligned}
$$

Proof We already know the continuity of $d_{\alpha}$ and that $d_{\alpha}(T)=0$. Moreover, from [5, p. 21, formula 1.2.2.19], $B^{*} P_{\alpha} \in \mathcal{L}(X ; C([0, T] ; U))$.

Recall that $d_{\alpha}(t)=p_{\alpha}(t)-P_{\alpha}(t) w_{\alpha}(t)$. First let us treat $p_{\alpha}$. Using the Hamilton equation (10), we see that

$$
\frac{d}{d t}\left\langle x, p_{\alpha}(t)\right\rangle=-\left\langle C^{*} C x, w_{\alpha}(t)\right\rangle-\left\langle A x, p_{\alpha}(t)\right\rangle+\langle C x, y(t)\rangle .
$$

Now we consider differentiability of $P_{\alpha}(t)$. We use formula $[5,(1.2 .2 .14)]$ and Lemma 9 in order to compute

$$
\frac{d}{d t}\left\langle x, P_{\alpha}(t) w_{\alpha}(t)\right\rangle=\frac{d}{d r}\left\langle x, P_{\alpha}(r) w_{\alpha}(t)\right\rangle_{\mid r=t}+\frac{d}{d r}\left\langle x, P_{\alpha}(t) w_{\alpha}(r)\right\rangle_{\left.\right|_{r=t}=t} .
$$

The first addendum is

$$
\left\langle-A^{*} P_{\alpha}(t) x-P_{\alpha}(t) A x-C^{*} C x+\frac{1}{\alpha} P_{\alpha}(t) B B^{*} P_{\alpha}(t) x, w_{\alpha}(t)\right\rangle .
$$

The second addendum is computed from Lemma 9 (recall that $P_{\alpha}(t) x \in$ dom $A^{*}$ since $x \in \operatorname{dom} A$, see [5, property vii), p. 20]). We get

$$
\begin{aligned}
& \left\langle\left(-A^{*}\right)^{\gamma} P_{\alpha}(t) x, D v_{\alpha}(t)\right\rangle+\left\langle A^{*} P_{\alpha}(t) x, w_{\alpha}(t)\right\rangle \\
& =-\frac{1}{\alpha}\left\langle B^{*} P_{\alpha}(t) x, B^{*} p_{\alpha}(t)\right\rangle+\left\langle A^{*} P_{\alpha}(t) x, w_{\alpha}(t)\right\rangle .
\end{aligned}
$$

Now we subtract (17) and (18) from (16). The result follows.

Now we improve our information on the regularity of $d_{\alpha}(t)$ : 
THEOREM 12 We have $d_{\alpha} \in C^{1-\theta}\left([0, T] ; \operatorname{dom}\left(-A^{*}\right)^{\theta}\right)$, for every $0<$ $\theta<1$, and $d_{\alpha} \in L^{2}\left(0, T ; \operatorname{dom} A^{*}\right)$.

Proof We derive an integral representation formula for $d_{\alpha}$, which displays the desired regularity properties. For any $s \in[0, T]$, set $\left[P_{\alpha}(s) B\right]=$ $\left[B^{*} P_{\alpha}(s)\right]^{*}$. From $[5$, p. 21$]$ it follows that $P_{\alpha} B$ is linear and continuous from $U$ to $C([0, T] ; X)$. Moreover, $B^{*} d_{\alpha}(s)=B^{*} p_{\alpha}(s)-B^{*} P_{\alpha}(s) w_{\alpha}(s)$ is well defined and continuous, the first addendum from Lemma 3 and the second one from the continuity of $w_{\alpha}(s)$ and of $B^{*} P_{\alpha}(s)$. For the same reason, $s \rightarrow\left[P_{\alpha}(s) B\right] B^{*} d_{\alpha}(s)$ is continuous so that, from (15), the following representation formula holds:

$$
\begin{aligned}
& d_{\alpha}(t)=-\int_{t}^{T} e^{A^{*}(s-t)}\left\{\frac{1}{\alpha}\left[P_{\alpha}(s) B\right] B^{*} d_{\alpha}(s)+C^{*} y(s)\right\} d s \\
& =\int_{t}^{T} e^{A^{*}(s-t)} f(s) d s,
\end{aligned}
$$

where $f(s)$ is bounded on $[0, T]$ with values in $X$. Now we conclude as in Lemma 6.

We are going to prove a variation of constants formula for $d_{\alpha}(t)$. Namely, we want to prove

THEOREM 13 The function $d_{\alpha}(t)$ is given by

$$
d_{\alpha}(t)=-\int_{t}^{T} U(T-t, T-s) C^{*} y(s) d s
$$

where $U(t, s)$ is an evolution operator which is exponentially bounded, strongly continuous and which transforms $X$ into $\operatorname{dom} B^{*}=\operatorname{dom}\left(-A^{*}\right)^{\gamma}$, for a.e. $t>s$.

In order to reduce the notation to a more usual form, it is convenient to replace $\xi(t)=d_{\alpha}(T-t)$. A simple transformation shows that $\xi(t)$ solves

$$
\xi(t)=\int_{0}^{t} e^{A^{*}(t-r)}\left\{[\tilde{P}(r) B] B^{*} \xi(r)-C^{*} \tilde{y}(r)\right\} d r
$$

where $\tilde{P}(r)=-\frac{1}{\alpha} P_{\alpha}(T-r), \tilde{y}(r)=y(T-r)$. To prove the previous theorem we need the next result.

THEOREM 14 There exists a unique strongly continuous and exponentially bounded evolution family $U(t, s)$ which, for $t>s$, is defined by

$$
U(t, s) x=e^{A^{*}(t-s)} x+\int_{s}^{t} U(t, r)[\tilde{P}(r) B] B^{*} e^{A^{*}(r-s)} x d r,
$$


for every $x \in X$. Moreover, for a.e. $t>s$, we have $U(t, s) X \subseteq \operatorname{dom} B^{*}$ and $[\tilde{P}(\cdot) B] B^{*} U(\cdot, s) x$ is locally integrable on $[s,+\infty)$, for every $x \in X$. The evolution family $U(t, s)$ verifies, for $t \geq s$ and $x \in X$,

$$
U(t, s) x=e^{A^{*}(t-s)} x+\int_{s}^{t} e^{A^{*}(t-r)}[\tilde{P}(r) B] B^{*} U(r, s) x d r .
$$

Proof We use Theorem 9.19, p. 487, in [2]. Let $U_{0}(t, s)=e^{A^{*}(t-s)}$ and let $\mathcal{B}(t)=[\tilde{P}(t) B] B^{*}$. Then, $\operatorname{dom} \mathcal{B}(t)=\operatorname{dom} B^{*}$ is constant and we have $\mathcal{B}(\cdot) U_{0}(\cdot, s)$ strongly continuous for $t>s$, with

$$
\left\|\mathcal{B}(t) U_{0}(t-s)\right\|=\left\|[\tilde{P}(t) B] B^{*} e^{A^{*}(t-s)}\right\| \leq \frac{M}{(t-s)^{\gamma}},
$$

locally integrable, from (3). The conclusion follows from this.

Proof of Theorem 13. We introduce

$$
\tilde{\xi}(t)=-\int_{0}^{t} U(t, s) C^{*} \tilde{y}(s) d s .
$$

We are going to prove that $\xi(t)=\tilde{\xi}(t)$. We see from (19) that $s \rightarrow$ $B^{*} U(t, s) x$ is integrable on $[0, t]$, for any $x \in X$. Since $B^{*}$ is closed, it is straightforward to check, by using suitable Riemann sums, that

$$
B^{*} \tilde{\xi}(t)=-\int_{0}^{t} B^{*} U(t, s) C^{*} \tilde{y}(s) d s .
$$

Moreover, we see from (19)

$$
\tilde{\xi}(t)=-\int_{0}^{t} e^{A^{*}(t-s)} C^{*} \tilde{y}(s) d s+\int_{0}^{t} e^{A^{*}(t-s)}[\tilde{P}(s) B] B^{*} \tilde{\xi}(r) d r .
$$

Hence, $\xi(t)$ and $\tilde{\xi}(t)$ solve the same Volterra integral equation, and

$$
[\xi(t)-\tilde{\xi}(t)]=\int_{0}^{t} e^{A^{*}(t-r)}[\tilde{P}(r) B] B^{*}[\xi(r)-\tilde{\xi}(r)] d r
$$

so that also

$$
\left(B^{*}[\xi(t)-\tilde{\xi}(t)]\right)=\int_{0}^{t} B^{*} e^{A^{*}(t-r)}[\tilde{P}(r) B]\left(B^{*}[\xi(r)-\tilde{\xi}(r)]\right) d r .
$$

Thanks to the inequality (3), Young inequalities and continuity of

$$
[\tilde{P}(r) B]
$$


the operator on $L^{2}(0, \tilde{T})$ defined by

$$
\phi(\cdot) \longrightarrow \int_{0}^{t} B^{*} e^{A^{*}(t-r)}[\tilde{P}(r) B] \phi(r) d r
$$

has norm less then $M T^{\gamma}$ for a suitable number $M$; so it is a contraction on $L^{2}\left(0, T_{1}\right), T_{1}<(1 / M)^{1 / \gamma}=T_{1}$. Hence $B^{*}[\xi(t)-\tilde{\xi}(t)]$ is zero on $\left[0, T_{1}\right]$ and, for $t>T_{1}$,

$$
\left.\left(B^{*}[\xi(t)-\tilde{\xi}(t)]\right)=\int_{T_{1}}^{t} B^{*} e^{A^{*}(t-r)} \tilde{[} P(r) B\right]\left(B^{*}[\xi(r)-\tilde{\xi}(r)]\right) d r .
$$

The same argument shows that $\left(B^{*}[\xi(t)-\tilde{\xi}(t)]\right)$ is zero on $\left[T_{1}, 2 T_{1}\right]$ too. In fact, it is easily seen that the norm of the operator

$$
\phi(\cdot) \longrightarrow \int_{T_{1}}^{t} e^{A^{*}(t-r)}[\tilde{P}(r) B] \phi(r) d r
$$

from $L^{2}\left(T_{1}, 2 T_{1}\right)$ in itself, is less then $M T_{1}^{\gamma}$, with the same coefficient $M$ as above. After a finite number of steps we see that $B^{*}[\xi(t)-\tilde{\xi}(t)]$ is zero on $[0, T]$ so that, from $(20)$ we have $\xi(t)=\tilde{\xi}(t)$ on $[0, T]$. This finishes the proof.

\section{References}

[1] A. Bensoussan, G. Da Prato, M. C. Delfour, and S. K. Mitter. Representation and control of infinite-dimensional systems. Birkhäuser, Boston, 1992.

[2] K-J. Engel and R. Nagel. One-parameter semigroups for linear evolution systems. Springer-Verlag, Berlin, 2000.

[3] J. M. Grimble and M. A. Johnson. Optimal control and stochastic estimation: theory and applications. John Wiley \& Sons, Chichester, 1988.

[4] I. Lasiecka, L. Pandolfi, and R. Triggiani. A singular control approach to highly damped second-order abstract equations and applications. Applied Mathematics 8 Optimization, 36:67-107, 1997.

[5] I. Lasiescka and R. Triggiani. Control theory for partial differential equations: continuous and approximation theories: Abstract parabolic systems. Cambridge University Press, cambridge, 2000.

[6] A. Lunardi. Analytic Semigroups and Optimal Regularity in Parabolic Problems. Birkhäuser, Basel, 1995.

[7] A. Pazy. Semigroups of linear operators and applications to partial differential equations. Springre-Verlag, Berlin, 1983.

[8] E. D. Sontag. Mathematical Control Theory. Springer-Verlag, Berlin, 1990. 